\title{
Content based image retrival using invariant color and texture features
}

\author{
K.Chaitanya Sruthi ${ }^{1}$, Shaik.Peer Ahamed ${ }^{2}$ \\ M.Tech [Scholar], Dept. of ECE, Chaitanya Engineering college, Visakhapatnam, AP, India ${ }^{1}$ \\ Assistant Professor, Dept. of ECE, Chaitanya Engineering college, Visakhapatnam, AP, India ${ }^{2}$
}

\begin{abstract}
Content Based Image Retrieval (CBIR) techniques appeared in 1990s. It uses low-level features like color, texture and shape to describe image content, and breaks through the limitation of traditional text query technique. This project we are proposes an image retrieval method based on color-feature and texture-feature similarity score. Many methods can be used to describe color feature. In this project we will use color moment method because it has the lowest feature vector dimensions and lower computational complexity. The HSV color space is describes a specific color by it's hue, saturation, and brightness values. For the similarity measurement the first order mean, the second standard deviation, and the third skewness color moments have been provided to be efficient and effective in representing color distribution of images. In this paper, the retrieval results from color feature and texture feature are analyzed, furthermore texture can be through as repeated patterns of pixel over a spatial domain. Since there is no mathematical definition for texture, many different methods are proposed for computing texture. Here we are used different distance based similarity measurement methods are proposed, before we are doing texture features apply ranklet transform can be calculated at different resolutions using Haar wavelet supports.
\end{abstract}

Keywords: Smart antenna; wireless mobile network; adaptive antenna; broadcast scheduling.

\section{I.INTRODUCTION}

Color is one of the most reliable used low level visual features and is invariant to image size and orientation. The use of low level visual features is to retrieve relevant information from image databases. The MPEG-7 consists of number of histogram descriptors and dominant color descriptors[1-5]. MPEG-7 specifies seven color descriptors. It consists of color space, color quantization, dominant colors, scalable color histogram, color structure, color layout and GOF/GOP color. The dominant colors are used to reduces the quality of image content. In this paper, we will implement an effective representative color quantization algorithm and improve the similarity measure for DCD. The DCD contains two main components, they are

\section{Representative colors and}

2. Their percentages in the image or region.

Texture is another important visual feature that has been intensively studied in pattern recognition. It refers the surface properties of an object and their relationship to the surrounding environment. Texture consists of some basic primitives, and also describes the structural arrangement of a region and the relationship of the surrounding regions. Texture features can be classified into two categories, firstly spectral features such as Gabor filter and discrete wavelet transformation. Secondly, statistical features such as wold feature tamura feature and gray level co occurrence matrix representation.

Shape features can also provide powerful information for content based image retrieval. Humans can recognize objects solely from their shapes. The shape features are

different from other elementary visual features, like color or texture features. The shape features[8] can be classified into two categories, firstly boundary based and secondly region based.Invariant moments are then used to record the shape features.

The some applications of the content based image retrieval are Fingerprint identification, crime prevention, biodiversity information systems, digital libraries, historical research, fashion and graphic design, publishing, advertising and medicine.

The basic difference between textual and visual information is the nature of retrieval process. The retrieval of textual information is based on discovering semantic similarity between textual entities. The visual information retrieval is based on discovering perceptual similarity.

The concept of perceptual similarity is made by examining the type of queries that users to use when retrieving images from the image databases. The fig 1 represents the Block diagram of the content based image retrieval (CBIR) [6-10].

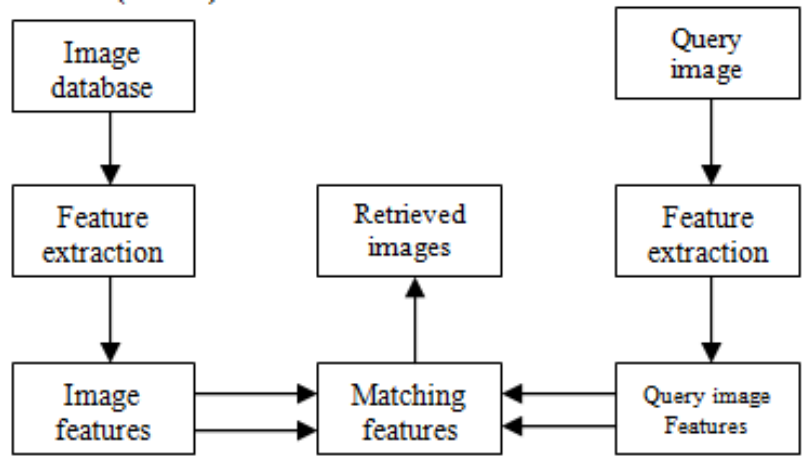

Fig.1. Block diagram of CBIR system.

rontent Based Image Retrieval (CBIR) is the retrieval of nages based on their visual features such as colour, :xture, and shape. Content-based image retrieval systems 
have become a reliable tool for many image database applications. There are several advantages of image retrieval techniques compared to other simple retrieval approaches such as text based retrieval techniques. CBIR provides a solution for many types of image information management systems such as medical imagery, criminology, and satellite imagery. In this computer age, virtually all spheres of human life including commerce, government, academics, hospitals, crime prevention, surveillance, engineering, architecture, journalism, fashion and graphic design, and historical research use images for efficient services. A large collection of images is referred to as image database. An image database is a system where image data are integrated and stored. Image data include the raw images and information extracted from images by automated or computer assisted image analysis. Image retrieval based on content is extremely useful in a plethora of applications such as publishing and advertising, historical research, fashion and graphic design, architectural and engineering design, crime prevention, medical diagnosis, geographical information and remote sensing systems, etc.. A typical image retrieval application example is a design engineer who needs to search his organization database for design projects similar to that required by his clients, or the police seeking to confirm the face of a suspected criminal among faces in the database of renowned criminals. In the commerce department, before trademark is finally approved for use, there is need to find out if such or similar ones ever existed. In hospitals, some ailments require the medical practitioner to search and review similar X-rays or scanned images of a patient before proffering a solution. A typical CBIR uses the contents of an image to represent and access. CBIR systems extract features (color, texture, and shape) from images in the database based on the value of the image pixels. These features are smaller than the image size and stored in a database called feature database. Thus the feature database contains an abstraction (compact form) of the images in the image database; each image is represented by a compact representation of its contents (color, texture, shape, and spatial information) in the form of a fixed length realvalued multicomponent feature vectors or signature. This is called offline feature extraction. The main advantage of using CBIR system is that the system uses image features instead of using the image itself. So, CBIR is cheap, fast, and efficient over image search methods. A key component of the CBIR system is feature extraction. A feature is a characteristic that can capture a certain visual property of the image. CBIR differs from classical information retrieval in that the image databases are essentially unstructured, since digitized images consist purely of arrays of pixel intensities, with no inherent meaning. One of the key issues with any kind of image processing is the need to extract useful information from the raw data (such as recognizing the presence of particular shapes or textures) before any kind of reasoning about the image's contents is possible. Early studies on CBIR used a single visual content such as color, texture, or shape to describe the image. The drawback of this method is that using one feature is not enough to describe the image since the image contains various visual characteristics. In this paper, we propose to extract both color and texture features from the image. Color and texture feature extract are simpler compared to other features.

\section{Color and Texture based similarity calculations}

Color is the sensation caused by the light as it interacts with our eyes and brain [11-13]. Color features are the fundamental characteristics of the content of images. Human eyes are sensitive to colors, and color features enable human to distinguish between objects in the images. Colors are used in image processing because they provide powerful descriptors that can be used to identify and extract objects from a scene. Color features provide sometimes powerful information about images, and they are very useful for image retrieval. Many methods [11-15] can be used to describe color feature. There are color histogram, color correlation, color moments, color structure descriptor (CSD), and scalable color descriptor (SCD). In this paper, we will use color moment method because it has the lowest feature vector dimension and lower computational complexity. To extract the color features from the content of an image, we need to select a color space and use its properties in the extraction. In common, colors are defined in three-dimensional color space. In digital image purposes, RGB color space is the most prevalent choice. The main drawback of the RGB color space is that it is perceptually non-uniform and device dependent system. The HSV color space is an intuitive system, which describes a specific color by its hue, saturation, and brightness values. This color system is very useful in interactive color selection and manipulation. The first-order (mean), the second (standard deviation), and the third-order (skewness) color moments have been proved to be efficient and effective in representing color distributions of images. If the value of the $i$ th color channel at the $\mathrm{j}$ th image pixel is pi $\mathrm{j}$, then the color moments are as follows.

\section{Similarity Measurement:}

a) Energy:The energy measure tells us something about how the intensity levels are distributed

b) Entropy:The entropy is a measure that tells us how many bits we need to code the image data

c) Standard deviation:The standard deviation, which is also known as the square root of the variance, tells us something about the contrast

d) Probability

e) Skew 
Moment 1: Mean

$E_{i}=\frac{1}{N} \sum_{j=1}^{N} p_{i j}$

Moment 2: Standard Deviation

$\sigma_{i}=\sqrt{\left(\frac{1}{N} \sum_{j=1}^{N}\left(p_{i j}-E_{i}\right)^{2}\right)}$

Moment 3: Skewness

$s_{i}=\sqrt[3]{\left(\frac{1}{N} \sum_{j=1}^{N}\left(p_{i j}-E_{i}\right)^{3}\right)}$

The color feature similarity measure is given by

$$
S_{\text {color }}(Q, I)=\sum_{i=1}^{N_{Q}} \sum_{j=1}^{N_{I}} d_{i, j} S_{i j j}
$$

Where $\mathrm{N}_{\mathrm{Q}}$ and $\mathrm{N}_{\mathrm{I}}$ denotes the number of dominant colors of the query image $\mathrm{Q}$ and the target image I respectively.

When retrieving the images we calculate the similarity between the query image and each target image in the image database, and then sort the retrieval results according to the similar value using Distance measurement Method :

$$
\begin{aligned}
& \text { - } \\
& \text { - } \quad \operatorname{Suclidean~Distance~}\left(\left(\mathrm{X}_{2}-\mathrm{X}_{1}\right)^{2}+\left(\mathrm{Y}_{2}-\mathrm{Y}_{1}\right)^{2}\right) \\
& \text { Manhattan Distance }
\end{aligned}
$$

Take the sum of the absolute values of the differences of the coordinates. For example, if

$\mathrm{X}=(\mathrm{a}, \mathrm{b})$ and $\mathrm{y}=(\mathrm{c}, \mathrm{d})$, the Manhatten distance between $\mathrm{x}$ and $\mathrm{y}$ is $|\mathrm{a}-\mathrm{c}|+|\mathrm{b}-\mathrm{d}|$.

$$
\begin{array}{r}
D_{M}(x)=\sqrt{(x-\mu)^{T} S^{-1}(x-\mu)} . \\
\text { - } \begin{array}{c}
\text { Mahalanobis Metric } \\
\text { Bray Curtis Distance }
\end{array} \\
\text { Minkowski Distance }=\left(\sum_{i=1}^{n}\left|x_{i}-y_{i}\right|^{p}\right)^{1 / \mathrm{p}}
\end{array}
$$

One fundamental step in CBIR system is the similarity measures. Similarity between two images is to find the distance between them. The distance between two images can be calculated using feature vectors that are extracted from the images. Therefore, the retrieval result is not a single image, but many images will be retrieved similar to the input image. Different similarity measures have been proposed based on the empirical estimates of the distribution of features, so the kind of features extracted from the image and the arrangement of these features in a vector will determine the kind of similarity measures to be used. Different similarity measures will affect the retrieval performance of image retrieval significantly.

Quantifying texture content of an image is the most important method to image region description. No formal definition for texture, but we can say that it provides the measures of properties such as smoothness, coarseness, and regularity. Furthermore, texture can be thought as repeated patterns of pixels over a spatial domain. If the texture has exposed to some noise, the patterns and their repetition in the texture can be random and unstructured. Since there is no accepted mathematical definition for texture, many different methods are proposed for computing texture. Among these methods, no single method works best with all types of texture.

Some common methods are used for texture feature extraction such as statistical, model-based, and transformbased methods. Before we extract the texture feature from the image, we perform a preprocessing step using Ranklet Transform. The result of applying Ranklet Transform on the image is 3 ranklet images in different orientation (vertical, horizontal, and diagonal).

Ranklet Transform belongs to a family of nonparametric, orientation-selective, and multiresolution features that has the wavelet style. It has been used for pattern recognition and in particular to face detection. Later on, it has been used for testing and estimating 3D structure and motion of objects.

From 2004, Ranklet Transform has been used in medical fields. It has been applied to the problems of tumoral masses detection in digital mammograms. Some tests show that Ranklet Transform performs better than some methods such as pixel-based and wavelet-based image representations.

\section{METHODOLOGY/FRAME WORK}

The methodology of the experimentation to retrieve images according to the query image is explained in this section.

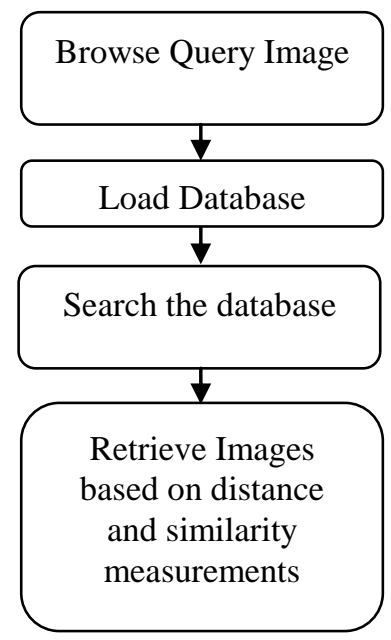

Fig.2: Frame work of the simulation

\section{SIMULATION RESULTS}

The simulation results pertaining to the proposed method are presented in the fig.3 (a) through 3(f). 


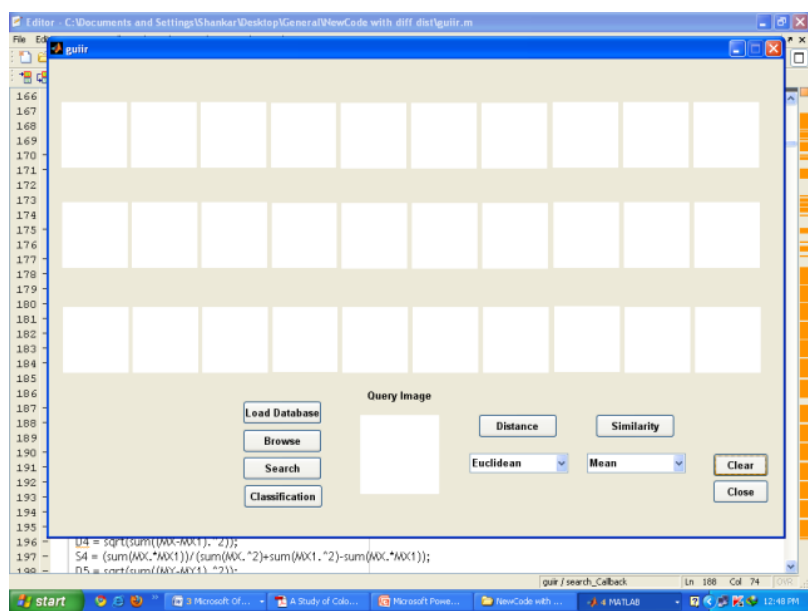

(a)

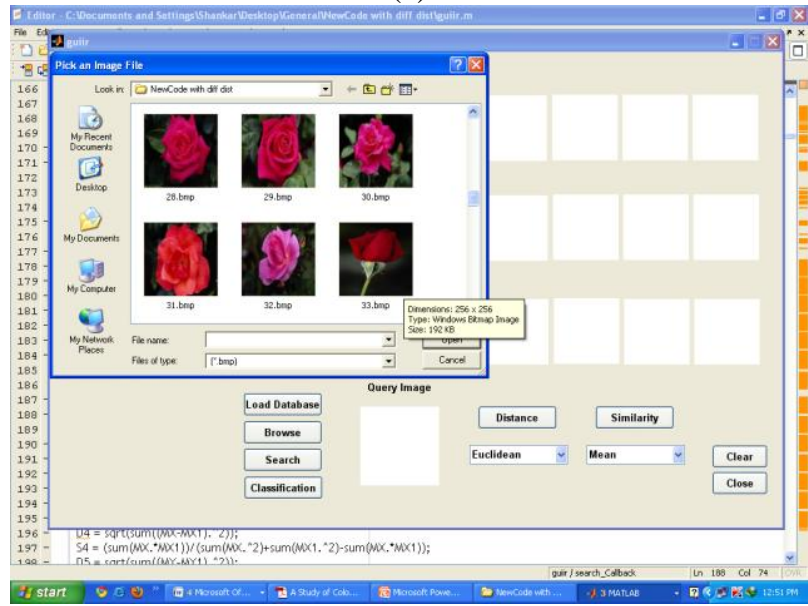

(b)

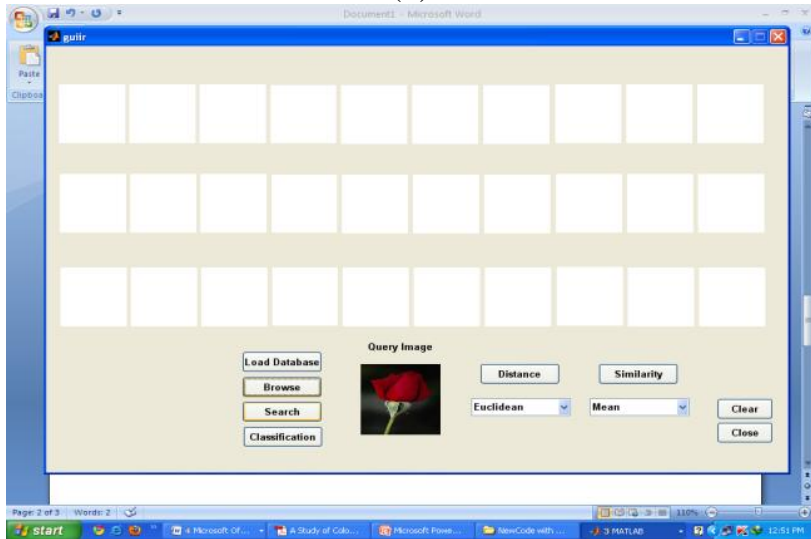

(c)

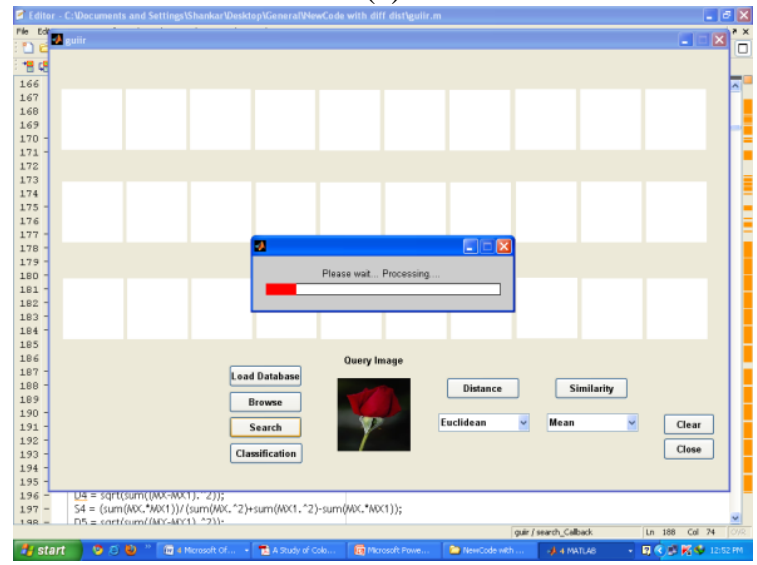

(d)

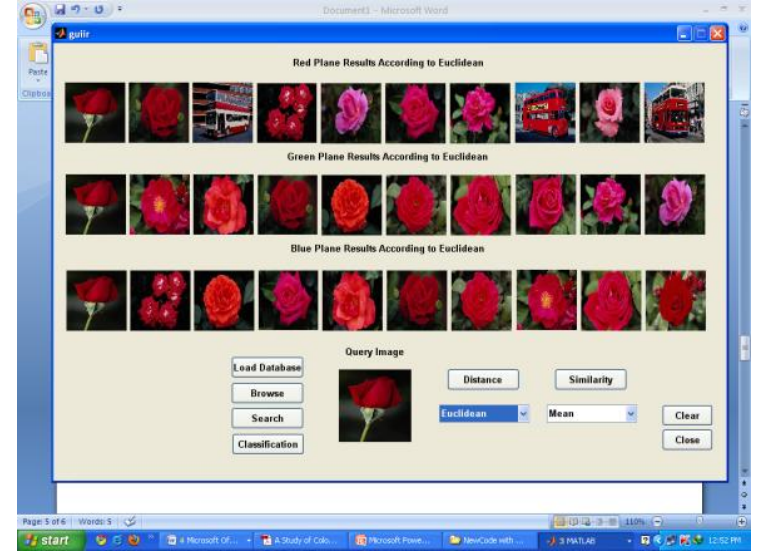

(e)

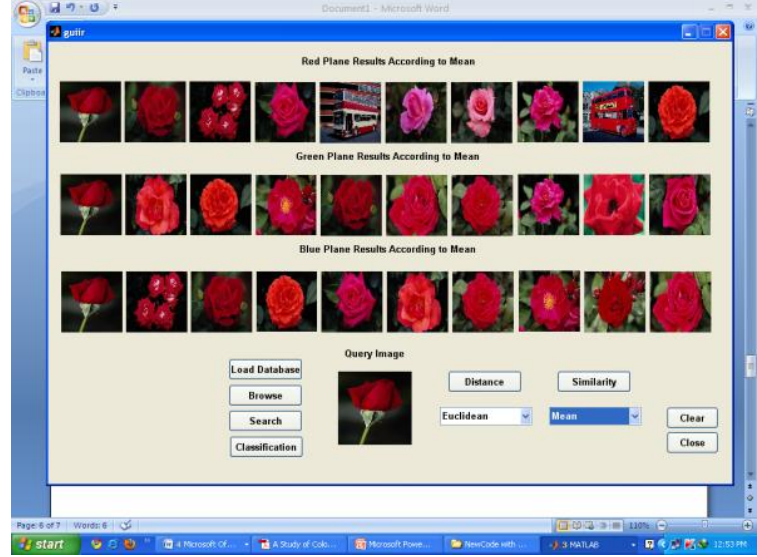

(f)

Fig.3: Simulation output screen shots

\section{IV.CONCLUSION}

Although CBIR has been a very active research area since 1990's, many challenges are issued because of the complexity of image data. Many researches have been done to develop some algorithms that solve some problems and achieve the accuracy when retrieving images and distinguishing between them. Many proposed algorithms use images to extract features and use their features for similarity matching. However, most of the algorithms use the gray scales images. Experimental results for ten class images showed that the proposed method has higher retrieval accuracy than those based on color and texture features respectively. Because the method uses multifeatures, which make use of each feature's unique advantages, in addition the dimensions of features vector are low. In the future, we propose a method that combines the shape and spatial features with the color and texture feature to represent the image. This will give good results. Also, segmentation is a method to extract regions and objects from the image. The segmented regions are used for similarity matching.

\section{REFERENCES}

[1] Barbeau Jerome, Vignes-Lebbe Regine, and Stamon Georges, "A Signature based on Delaunay Graph and Co-occurrence Matrix," Laboratoire Informatique et, Systematique, Universiyt of Paris, Paris, France, July 2002.

[2] Yousef Mahdy, Khaled Shaaban, and Ali Abd El-Rahim, "Image Retrieval Based on Content," GVIP Journal, Volume 6, Issue 1, July 2006. 
[3] Digital Systems \& Media Computing Laboratory,"Content-Based Image Retrieval," Hellenic Open University (HOU), GREECE, 2009. at ( http://dsmc.eap.gr/en/cbir.php).

[4] H. Tamura, and N. Yokoya, "Image Database Systems: A Survey," Pattern Recognition, vol. 17, no 1, pp.29-49, Sep. 1984.

[5] V. Gudivada and V. Raghavan, "Content-based image retrieval systems," IEEE Computer, vol. 28, no 9, pp18-22, Sep. 1995.

[6] F. Long, H. Zhang, H. Dagan, and D. Feng, "Fundamentals of content based image retrieval," in Multimedia Information Retrieval and Management, D. Feng, W. Siu, and H. Zhang, Eds., Multimedia Signal Processing Book, chapter 1, pp. 1-26, Springer, Berlin, Germany, 2003

[7] M. Flickner, H. Sawhney, W. Niblack, J. Ashley, Q. Huang, B. Dom, M. Gorkani, J. Hafner, D. Lee, D. Petkovic, and P. Yanker, "Query by image and video content:The QBIC system," IEEE Computer, vol. 28, no 9, pp.23-32, Sep. 1995.

[8] J. Smith and C. Li, "Image Classification and Querying Using Composite Region Templates," Int. J. Computer Vision and Image Understanding, vol. 75, no. 2, pp. 165-174, July 1999.

[9] A. Gupta, and R. Jain, "Visual information retrieval," Comm. Assoc. Comp. Mach., vol. 40, no. 5, pp. 70-79, May. 1997.

[10] S. Mukherjea, K. Hirata, and Y. Hara, "AMORE: A World Wide Web Imag Retrieval Engine," Proc. World Wide Web, vol. 2, no. 3, pp. 115- 132, June. 1999.

[11] A. Natsev, R. Rastogi, and K. Shim, "WALRUS: A Similarity Retrieval Algorithm for Image Databases," IEEE Trans. On Knowledge and Data Engineering, vol.16, pp. 301-318, Mar. 2004.

[12] A. Pentland, R. Picard, and S. Sclaroff, "Photobook: Content based manipulation of image databases," International Journal of Computer Vision, vol.18, no 3, pp.233-254, June 1997.

[13] C. Carson, M. Thomas, S. Belongie, J.M. Hellerstein, and J. Malik, "Blobworld: A System for Region-Based Image Indexing and Retrieval," Proc. Visual Information Systems, pp. 509-516, June 1999.

[14] J. Smith and S. Chang, "Visualseek: A Fully Automated ContentBased Image Query System," Proceedings of the 4th ACM international conference on Multimedia table of contents, Boston, Massachusetts, United States, Nov. 996, pp. 87-98.

[15] W. Ma and B. Manjunath, "Natra: A Toolbox for Navigating Large Image Databases," Proc. IEEE Int'l Conf. Image Processing, Santa Barbara, 1997, pp. 568-571. 